\title{
Twinned Epitaxy of Copper on Copper
}

\author{
Theodore H. Orem
}

\begin{abstract}
A study of the X-ray diffraction patterns of copper electrodeposited on copper monocrystals having surfaces parallel to the cubic, dodecahedral, and octahedral planes shows an interesting relationship between electrodeposit and base crystal. At the current density used for electrodeposition, the electrodeposit on the surface parallel to the dodecahedral plane was a monocrystalline continuation of the orientation of the base crystal in every respect. The electrodeposit on the surface parallel to the octahedral plane was a highly preferentially oriented polycrystalline electrodeposit made of microcrystals, some of which continued the orientation of the base crystal, whereas others were twinned with respect to the surface plane of the base erystal. In the case of electrodeposition on the surface parallel to the cubic plane, the electrodeposit was also a highly preferentially oriented polycrystalline electrodeposit made up of microcrystals, some of which continued the orientation of the base crystal, whereas others bore a twin relationship to the close-packed planes of the base erystal.
\end{abstract}

\section{Introduction}

A considerable number of investigators working in the field of electrodeposition have established that there exists a propensity for the crystallographic orientation of the electrodeposit to be the same as that of the basis metal, if favorable conditions of electrodeposition are maintained $[1,2,3,4,5] .{ }^{1} \quad \mathrm{~A}$ discussion based on these references is contained in Barrett's "Structure of Metals" [6]. This continuity of orientation (epitaxy) has been shown to be possible even though the substrate and electrodeposit are two different metals, provided the atomic radii of the two metals do not vary from each other by more than a certain critical percentage $[7,8,9]$. Takahashi [10] found an orientation relationship when a face-centered cubic metal, copper, was electrodeposited on beta brass, which is body-centered cubic.

In the course of an investigation designed to study the fundamental manner in which metals corrode, several specimens of monocrystalline copper were plated with copper in an effort to determine the orientation of the facets of etch pits that develop during the attack by a certain corrodent, in the hope that the plated facets, continuing the original orientation, would be more readily distinguishable than the original. X-ray diffraction patterns of the electrodeposited specimens showed slight but distinct differences compared to the diffraction patterns of similarly oriented unplated copper single crystals. The differences were considered significant enough to justify further investigation. Some of the results are described herein.

\section{Materials and Procedures}

A monocrystalline copper ingot, grown by the Bridgman method of slow cooling from the melt, was sectioned to produce specimens having surfaces approximately parallel to the cubic, dodecahedral, and octahedral planes. The specimens were mounted in Lucite, ground and polished, and then electropolished to remove cold work imparted during the mechanical

\footnotetext{
1 Figures in brackets indicate the literature references at the end of this paper.
}

grinding and polishing operations. Laue back-reflection diffraction patterns were obtained from each specimen, using unfiltered radiation from a cobalt target, in order to determine the degree in which the surface was parallel to the desired crystallographic plane. The specimens were then reground and repolished, when necessary, until the surfaces were within one-half degree of being parallel to the cubic, dodecahedral, or octahedral planes. Electrodeposition was carried out in an acid sulfate bath at a current density of approximately $30 \mathrm{ma} / \mathrm{cm}^{2}$. Electrolytic copper was used as the anode. Periods of plating ranged from a few minutes up to $18 \mathrm{hr}$. Backreflection diffraction patterns at normal incidence and using the same radiation as above were then obtained from the electrodeposited copper specimens. After analysis of the various diffraction patterns, the electrodeposits were electropolished, electroetched, and examined microscopically.

\section{Results and Discussion}

Figure 1 is a series of three photomicrographs showing the microstructures of the electrodeposits on the monocrystalline copper specimens having surfaces parallel to the cubic, dodecahedral, and octahedral planes. Two of the photomicrographs in figure 1 appear to be of polycrystalline metal, but figure $1, b$ is definitely monocrystalline in character. Figures 1, a and 1,c exhibit strong evidences of twinning in their microstructures, but figure $1, b$ shows no twinning.

\subsection{Deposition on Surface Parallel to an Octahedral Plane}

A photograph of a normal incidence Laue backreflection diffraction pattern obtained from a copper monocrystal whose surface is parallel to an octahedral plane is shown in figure 2,a. Figure 2,b is the same type of pattern from the same specimen after it had received an electrodeposit of copper. It is obvious that X-rays were reflected from more planes in the electrodeposited copper than were reflected from planes in the base crystal. 

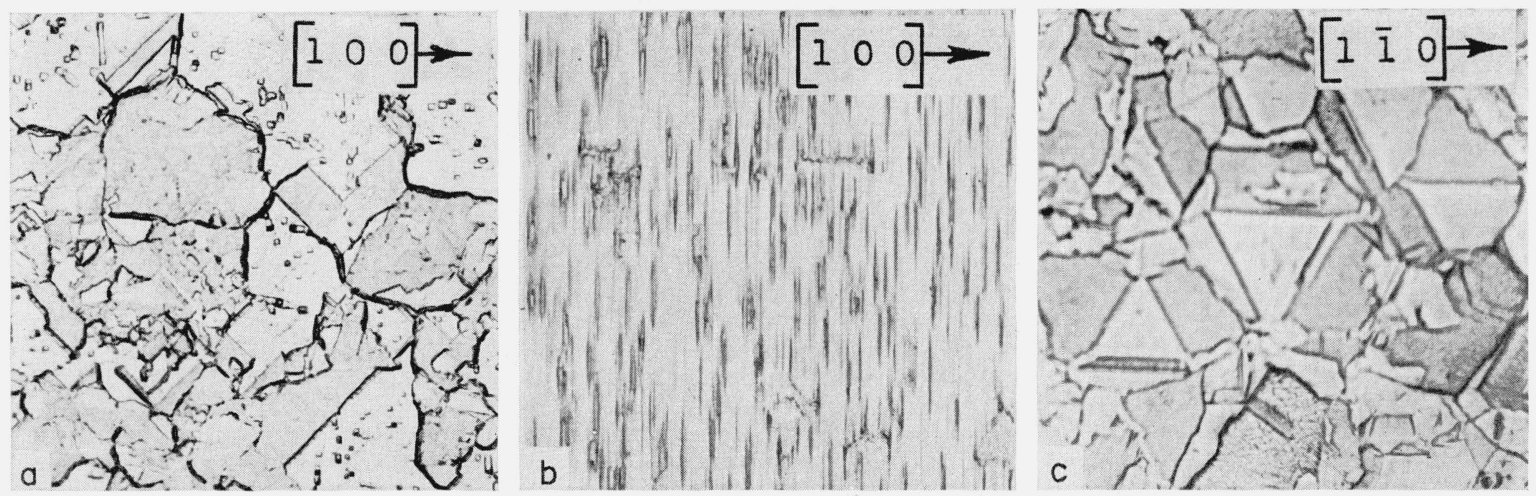

Figure 1. Photomicrographs of electropolished and electroetched copper electrodeposits on monocrystalline copper surfaces.

(a) Surface parallel to $\left(\begin{array}{lll}0 & 0 & 1\end{array}\right)$, (b) surface parallel to $\left(\begin{array}{lll}0 & 1 & 1\end{array}\right)$, and (c) surface parallel to (lll 111$)$. A principal direction in the surface of the base crystal is indicated on each photomicrograph. X 250 .

The normals of the reflecting planes represented by Laue spots in figure 2 , a were plotted in a stereographic triangle, as shown in figure 3 ,a. The normals of the reflecting planes represented by Laue spots in figure 2,b are shown in the stereographic triangle shown in figure 3,b. The solid circles represent normals of reflecting planes from which spots were obtained on both the unplated and plated specimens, and the open circles in figure $3, \mathrm{~b}$ are the normals to the reflecting planes represented by Laue spots obtained only from the plated specimen. The sizes of the circles are roughly proportional to the intensities of the pertinent Laue spots. Due to difficulty in photographically reproducing many of the less intense Laue spots present on the diffraction patterns used to obtain figure 2 , there is a seeming discrepancy between the number of Laue spots on these photographs and the number of corresponding poles indicated in figure 3. The latter, however, are the factual representation of the poles of the planes from. which reflections were obtained, as determined from the diffraction patterns. This same apparent discrepancy between spots on photographs of diffraction patterns and poles shown in corresponding stereographic triangles will be noted in the discussion of electrodeposition on surfaces parallel to the dodeca. hedral and cubic planes. In each instance the poles

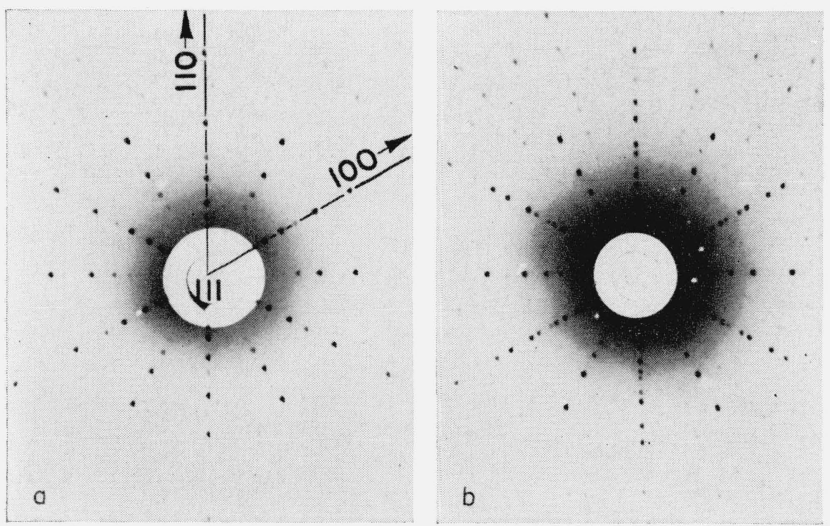

FIGURE 2. Laue back-reflection diffraction patterns.

(a) Before, and (b) after electrodeposition of copper in a copper monocrystal. The surface was parallel to an octahedral plane and the X-ray beam normal to the surface, shown in the stereographic triangles represent the Laue spots actually observed. The circles containing a cross will be explained later in a discussion of the means of indexing the various spots shown in figure 3.

The stereographic triangles shown in figure 3 are oriented so that the poles of the projection correspond to the spots in a segment of the diffraction patterns in figure 2. The segment is outlined only on the diffraction pattern from the base crystal, figure 2,a. The segment for the pattern in figure 2,b, is in the same relative position as that in figure 2 ,a because the azimuthal orientations of both patterns are the same. This same manner of presentation of

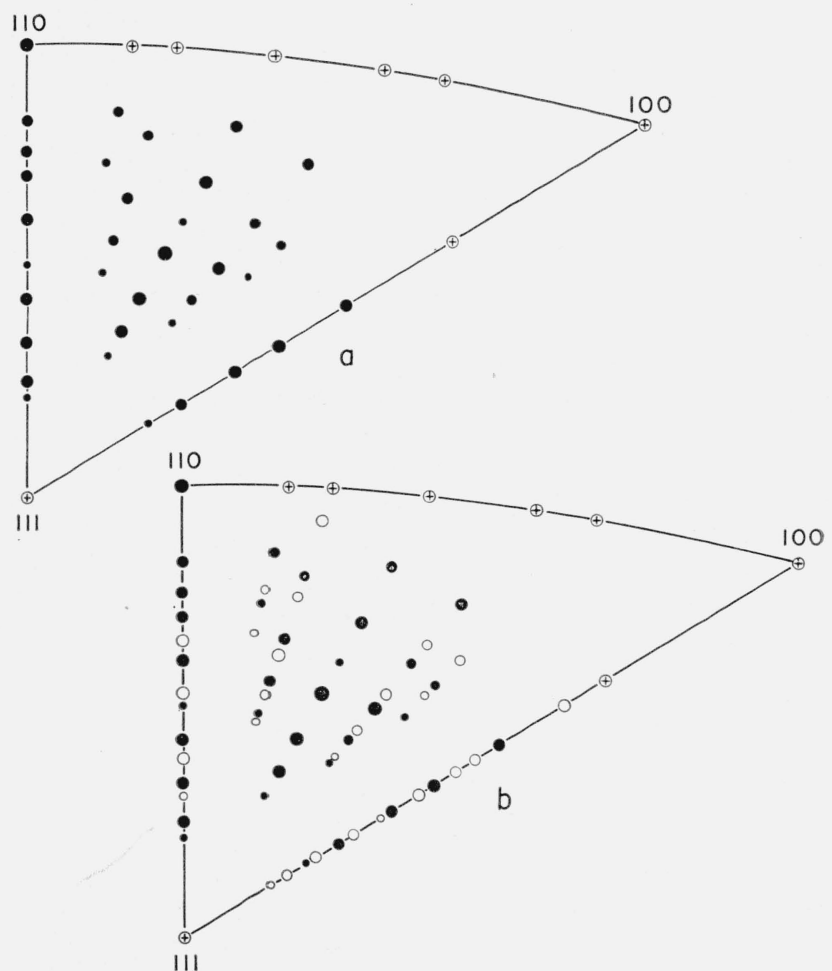

FIGURE 3. Unit stereographic triangles showing orientation of poles that correspond to the reflection spots in figure 2. 


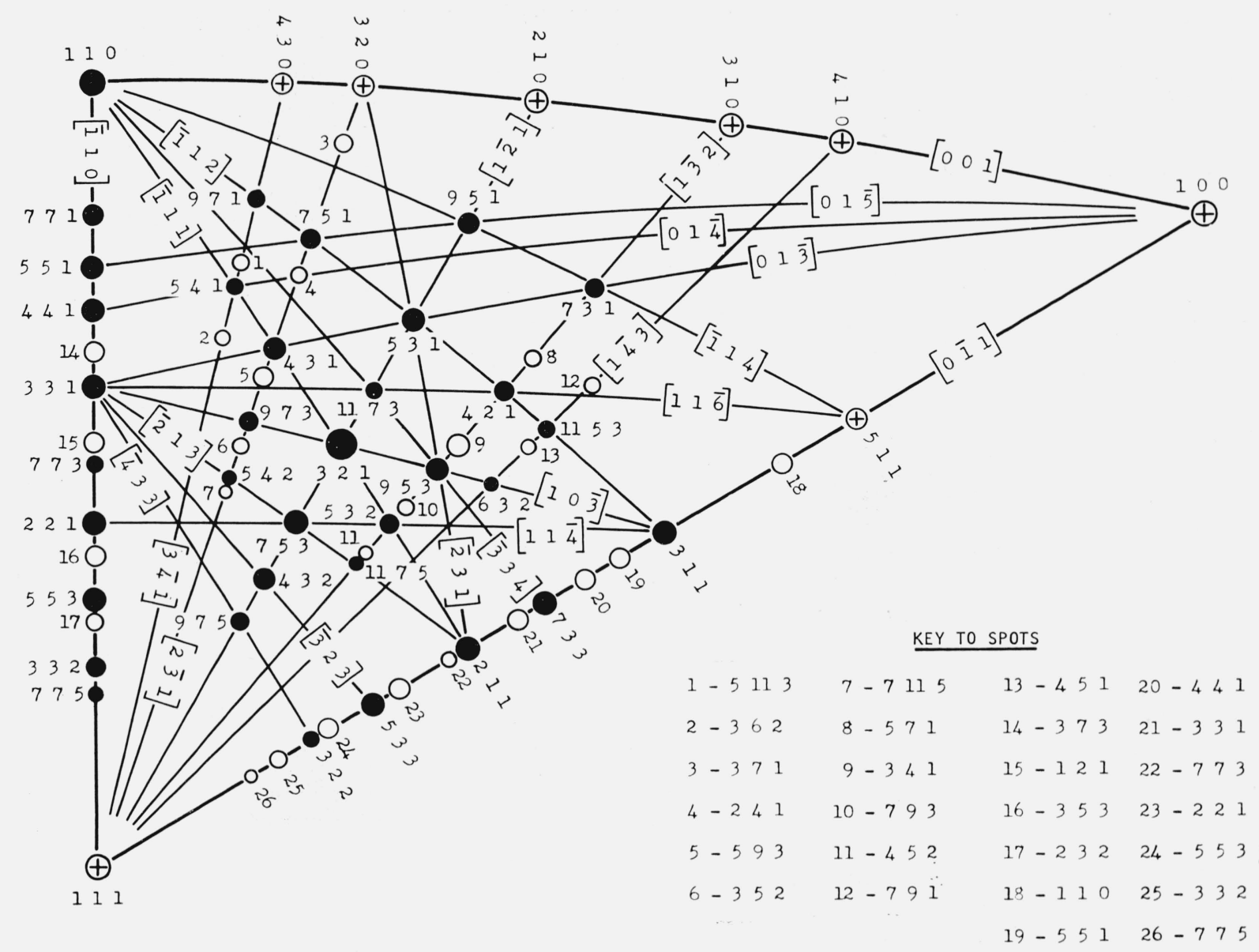

FIGURE 4. Stereographic triangle identifying spots and zones shown in figure 3.

stereographic triangles is followed throughout this paper.

Figure 4 is a stereographic triangle showing the spots shown in figure $3, \mathrm{~b}$. In this triangle it is necessary to know the location of certain poles, which could not be located from the pertinent diffraction pattern spots because of size limitations of the camera, in order to index certain zones that contain poles corresponding to Laue spots found in the diffraction pattern. These poles, not present as Laue spots on the diffraction pattern, have been located by means of circles containing crosses in order to distinguish them from all other poles in the triangle, which were actually located from Laue spots present in the diffraction pattern.

It will be noted that each of the solid circles in figure 4 is at the intersection of two or more zones and that the formulation of the indices of the spots represented by the solid circles is in accordance with the conventional crystallographic method for indexing such spots. The open-circle spots in figure $3, \mathrm{~b}$, indexed in figure 4 , are the location of poles corresponding to the Laue spots from the twin of the surface plane of the base crystal caused by a $60^{\circ}$ clockwise rotation about the octahedral axis parallel to the surface normal. An explanation of the method of indexing the open-circle spots is given in the appendix. An interpretation of figure 2,b, from which the position of the poles with respect to the octahedral pole in the stereographic triangle shown in figure 4 were determined, indicates that the twinning plane in the electrodeposit is parallel only to the octahedral plane parallel to the surface of the base crystal. At the current density used for this work, twinning occurred only on the surface plane. At other current densities it is conceivable that twinning might also occur on close-packed planes other than the surface plane.

A careful investigation of the spots from the twin orientation showed that, as electrodeposition progressed, they gradually increased in intensity to a value equal to that of the intensity of a corresponding spot in the base crystal. This would appear to indicate that a constant ratio between the twinned and untwinned orientations was built up in the electrodeposit.

The method of packing spheres to illustrate the regular and the twin position of an atom with respect to the uppermost two layers in the octahedral plane of the base crystal is shown in figure 5. Figure 5, a shows the atoms as they would be packed in the uppermost two layers of a monocrystalline specimen whose surface is parallel to an octahedral plane. Figure 5,b shows an atom (dark sphere) as it would be located in the first layer of the electrodeposit if 


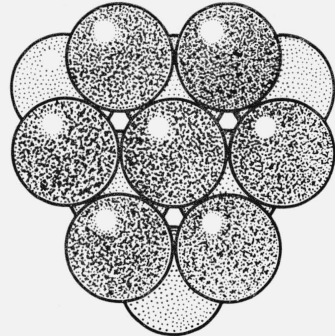

a
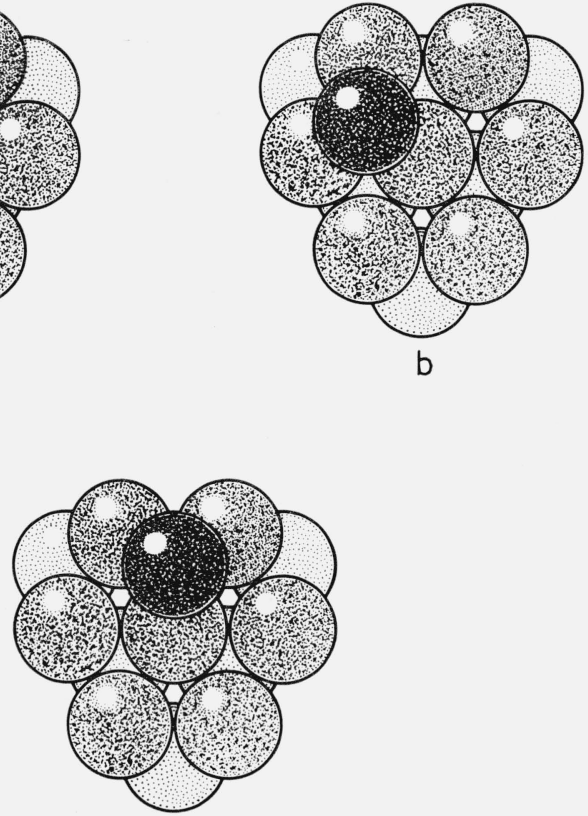

C

FIGURE 5. Sketches of packed spheres depicting the atomic arrangements in the octahedral plane of copper.

(a) The uppermost two layers in the octahedral plane, (b) the deposition of an atom of copper (dark sphere) in the first of two positions in which it might locate, and (c) the deposition of an atom of copper (dark sphere) in the second of two positions in which it might locate.

the deposition were such as to continue the atomic configuration of the basis metal, in kind. Figure 5 ,c shows the atom (dark sphere) located in the position that it would occupy if the deposition were such that the first layer deposited bore a twin relationship to the uppermost two layers of the base crystal.

Figure 6 shows by means of spheres the atomic arrangement at the interface of the base metal (bottom two layers) and electrodeposit (top layer) (a) if the atom were to locate as in figure $5, \mathrm{c}$, and (b) if the atom locates as in figure $5, \mathrm{~b}$. The arrangement of spheres in figure 6,a represents the atoms positioned in the $\mathrm{ABAB}$ arrangement of the hexagonal system, in which case the atoms of each alternate close-packed layer are arranged directly above one another. In the face-centered cubic system (copper) the fourth layer of close-packed atoms is directly over the first layer, the fifth layer directly over the second layer, the sixth over the
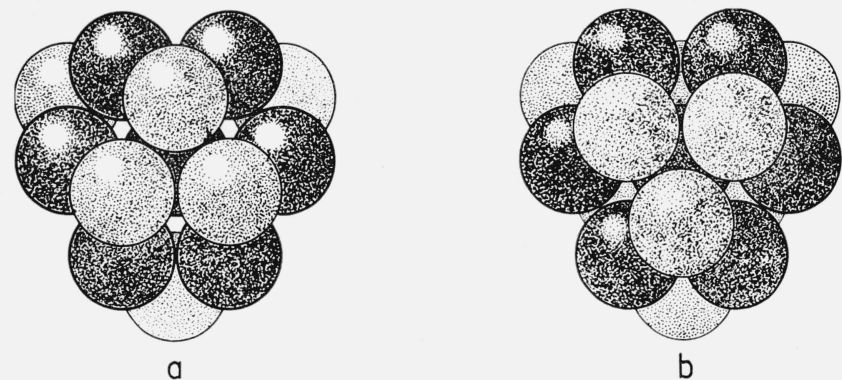

FIGURE 6. Sketches of packed spheres depicting the atomic arrangements at the interface of base crystal and electrodeposit.

(a) If copper atoms were to deposit at location shown in figure 5,c: (b) if copper atoms were to deposit at location shown in figure $5, \mathrm{~b}$. third, etc., in $\mathrm{ABCABC}$ fashion, as shown by the packing of spheres in figure $6, b$.

Judging from the microstructure of the electrodeposit shown in figure 1 ,c, it is apparent that a copper atom deposits from solution on the surface of the base crystal in either of the two positions shown in figures 5,b and 5,c, since the electrodeposit is made up of many small crystals, some of which have the same orientation as the base crystal, and some of which are twinned with respect to it, as shown by the diffraction pattern obtained from the electrodeposit.

It is suggested that when the copper atom deposits from solution it locates randomly on the original surface, either twinned or untwinned with respect to the base crystal. The originally deposited atoms are then nucleation points around which subsequently depositing atoms locate. These atoms probably deposit in monolayers around the nucleation atoms, those locating around the originally deposited twinned atom forming a twinned area and the atoms surrounding the untwinned first-deposited atom forming an untwinned monolayer of atoms. The two monolayers then grow laterally into each other. The juncture of a monolayer of twinned atoms and one of untwinned atoms is a line of misfit of atoms which constitutes a twin boundary. These lines of atom misfit are probably the sides of the equilateral triangles seen in figure 1,c. As subsequent monolayers of atoms deposit, the areas in which misfitting of atoms occurs eventually become large enough to preclude subsequent deposition in an orderly fashion and an irregularly formed grain boundary, such as may be observed in figure 1,c, results. As a consequence of this type of growth, the deposit is polycrystalline in character but with the individual crystals having a very high degree of preferred orientation. This structure is apparent both from the appearance of the microstructure, figure 1 ,c, and from the diffraction pattern of the electrodeposited crystal, figure 2,b.

\subsection{Deposition on Surface Parallel to Dodecahedral Plane}

Figure 7 ,a is a photograph of a Laue back-reflec-

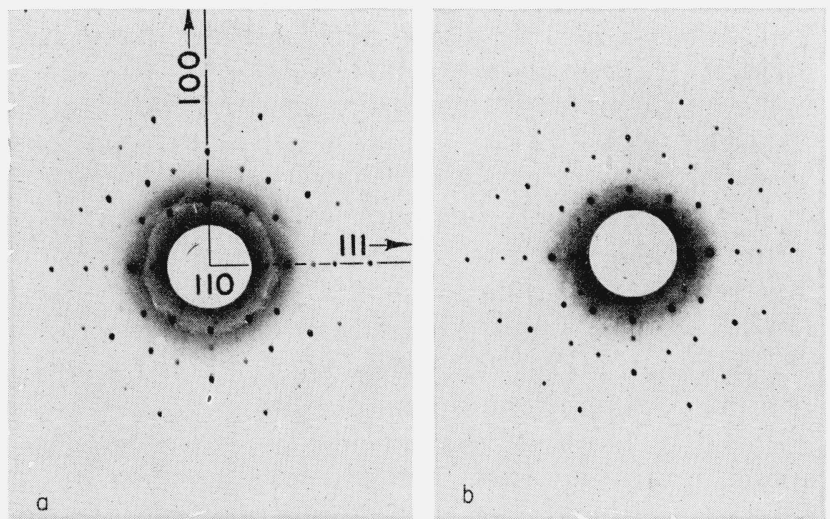

Figure 7. Laue back-reflection diffraction patterns.

(a) Before, and (b) after electrodeposition of copper on a copper monocrystal. The surface was parallel to a dodecahedral plane and the X-ray beam normal to the surface. 


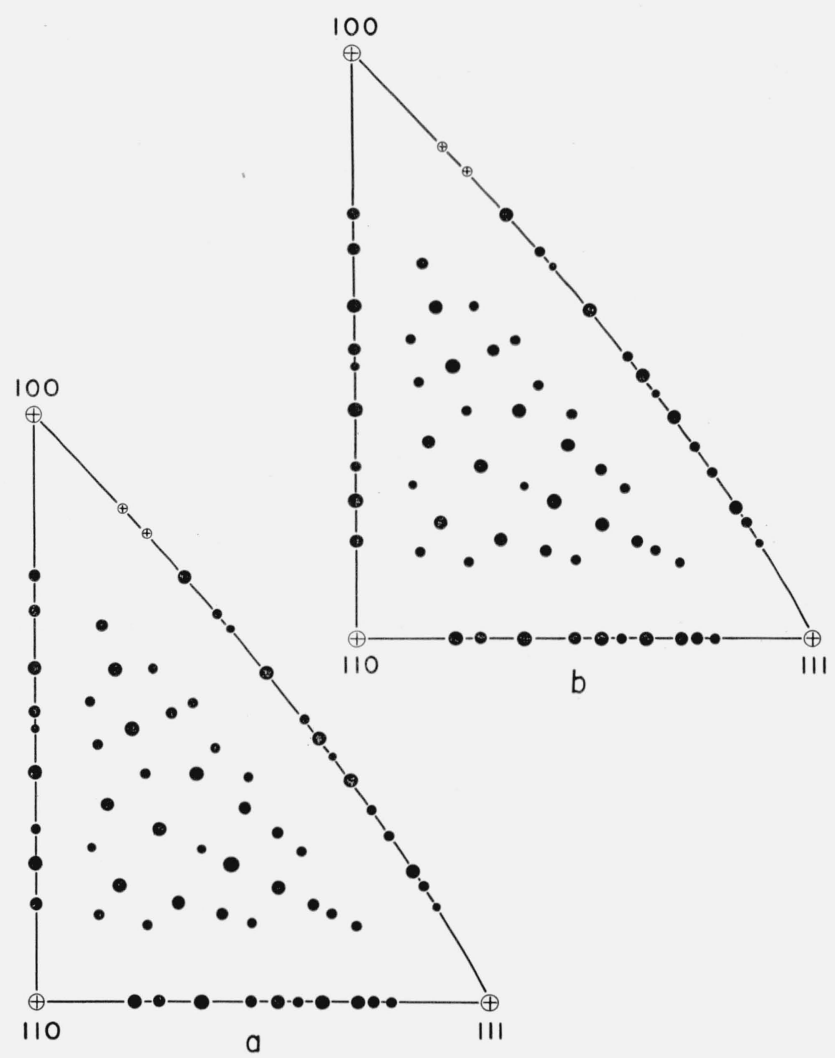

FIGURE 8. Unit stereographic triangles showing orientation of poles that correspond to the reflection spots in figure $\%$.

tion diffraction pattern of a monocrystalline copper specimen whose surface is parallel to the dodecahedral plane, the incident beam having been perpendicular to the specimen surface. Figure $7, b$ is a diffraction pattern of the same specimen after receiving a heavy deposit of copper.

Figures 8 ,a and $8, \mathrm{~b}$ are stereographic triangles representing the poles of reflecting planes from which Laue spots were obtained on the unplated and plated monocrystalline copper crystals shown in figure 7 . The solid and crossed circles represent poles, as previosuly described for figure 3 . It will be noted that there are no open circles in figure $8, b$, as there were in figure $3, \mathrm{~b}$, because reflections from planes other than those which caused reflections in the unplated specimen were not present on the diffraction pattern obtained from the electrodeposited specimen. Figure 9 is a stereographic triangle identifying the spots and zones shown in figure 8 .

The appearance of the microstructure of the electrodeposited specimen whose surface is parallel to the dodecahedral plane, figure 1,b, would appear to indicate that the atomic arrangement in the electrodeposit is little, if any, different from that in the base metal. There is no indication in the diffraction patterns of the base metal and the electrodeposited specimen of any difference in their orientations.

Figure 10 illustrates (a) the configuration of the uppermost two layers of atoms in a monocrystalline copper specimen whose surface is parallel to the dodecahedral plane, and (b) the positions the first layer of deposited atoms takes with respect to the layers on which it deposits. An atom depositing from solution on the surface shown in 10 , a may locate in any position on the surface and still preserve the atomic configuration of the substrate in so doing. Subsequently depositing atoms likewise do not alter the atomic configuration at the base crystal interface no matter where they deposit. Succeeding layers of atoms are deposited similarly, all in a very orderly fashion with respect to the underlying layers. Thus, when deposition is upon a surface parallel to a dodecahedral plane, the orientation of the atoms in the electrodeposit is exactly the same as that of the atoms in the base crystal. It is emphasized that such an electrodeposit was obtained at a specific current density. At other current densities it is conceivable that twinning might occur on this surface also.

\subsection{Deposition on Surface Parallel to a Cubic Plane}

Figure 11 , a is a photograph of a Laue back-reflection diffraction pattern obtained at normal incidence on a monocrystalline copper specimen whose surface is parallel to a cubic plane. Figure 11,b is the same kind of pattern obtained after the specimen has received an electrodeposit of copper. The difference in the number of Laue spots on the two photographs is obvious.

Figures 12 , a and 12,b are stereographic triangles whose poles correspond to the Laue spots shown in figure 11. The solid circles represent reflections obtained from both the unplated and plated specimens, and the open circles in figure $12, \mathrm{~b}$, represent additional spots found only in the diffraction pattern from the electrodeposited specimen. Figure 13 is a stereographic triangle identifying the spots and zones shown in figure 12 .

Comparing figures 12 , a and $12, \mathrm{~b}$, it is readily apparent that there are many more reflecting planes in the electrodeposit than there are in the base metal. Using figure 13 to identify the open-circle spots in figure $12, \mathrm{~b}$ it is obvious that the indices of reflections that were present only in the pattern from the electrodeposited copper were of a conspicuously higher order than the indices of reflections that were present in the pattern obtained from the base crystal.

Since the wavelengths, in accordance with Bragg's law, that correspond to the very high indices are smaller than the minimum wavelength used in making the patterns, it is obvious that the reflections are not from crystals having the same orientation as the base crystal. It is postulated that the apparently spurious reflections are from crystals that were formed by twinning on the octahedral planes of crystals that were deposited with an orientation corresponding to that of the base crystal. If this is true, then the twinning should result in the creation of certain low-index crystallographic planes in portions of the electrodeposit that are parallel to the high-index planes from which reflections have apparently been obtained. The high-index planes whose normals are shown in figure 13 were reindexed in accordance 


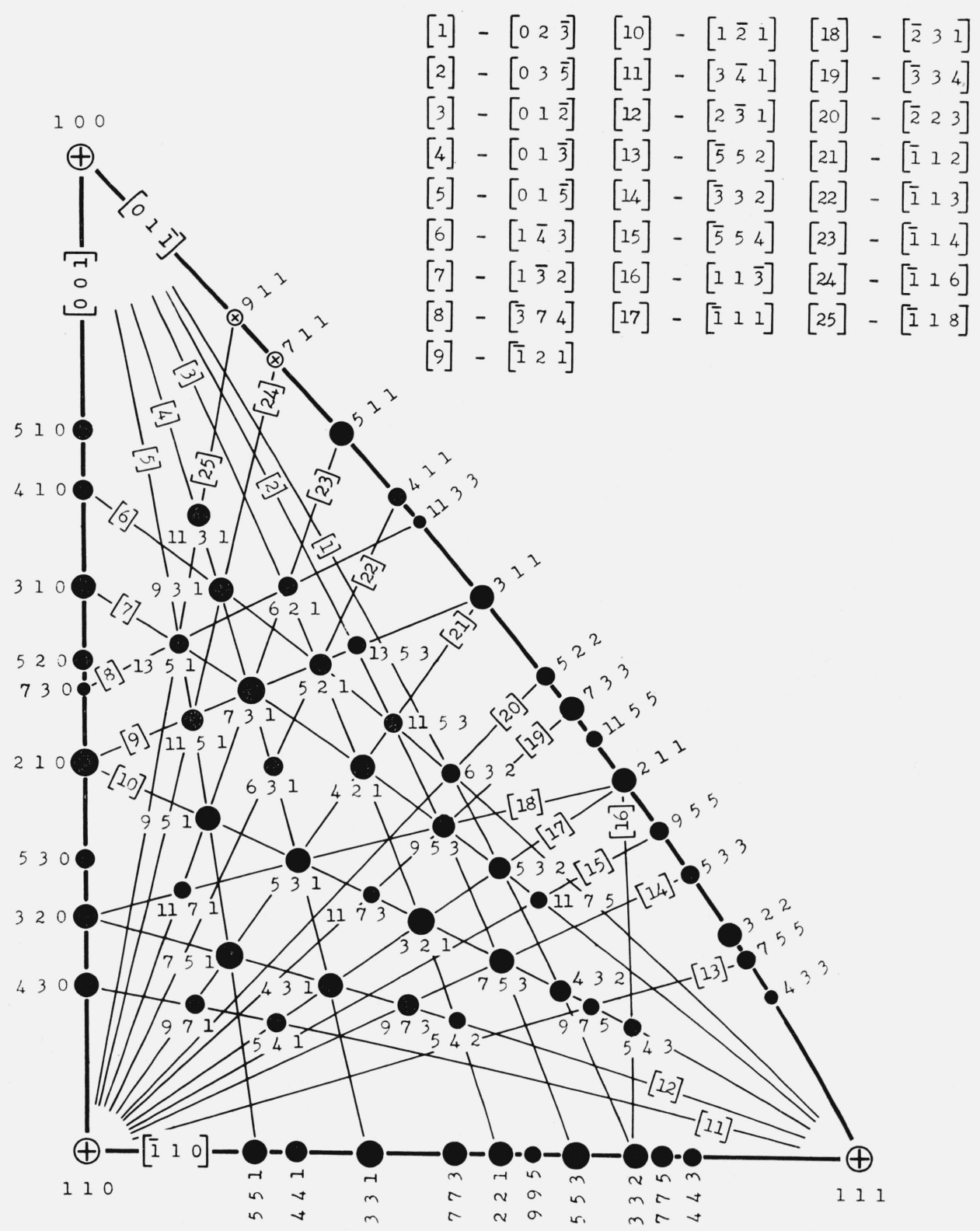

Figure 9. Stereographic triangle identifying spots and zones shown in figure 8.

with the method described in the appendix in an effort to determine whether the extra Laue spots seen in figure $11, \mathrm{~b}$ could be reflections from microcrystals in the electrodeposit bearing a twin relationship to the base crystal. Table 1 lists the reindexed planes as "true reflecting planes" opposite the planes as indexed in accordance with the orientation of the base crystal, which planes have been designated "apparent reflecting planes." The former are the low-index planes in the twinned portion of electrodeposit that are parallel to high-index planes in the base crystal and the untwinned portion of the electrodeposit. The apparently spurious reflections all now have indices with " $d$ " spacings that, in accordance with Bragg's law, correspond to wavelengths greater than the experimental minimum. Furthermore, the new indices are included in the families of indices obtained for the reflections in patterns of the base crystal. These facts justify the postulate that the electrodeposited copper consisted of crystals having orientations of the base crystal and its four twins.

Figure 14 illustrates by means of the packing-ofspheres method the atomic arrangements present in the preferentially oriented microcrystals making up the electrodeposit whose microstructure is shown in figure 1 ,a.

Figure 14 , a shows the atomic arrangement in the uppermost two layers when the surface is parallel to a cubic plane. Figure 14,b shows the position a depositing atom (dark sphere) will take with re- 


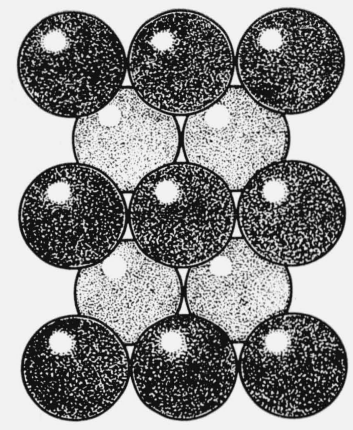

a

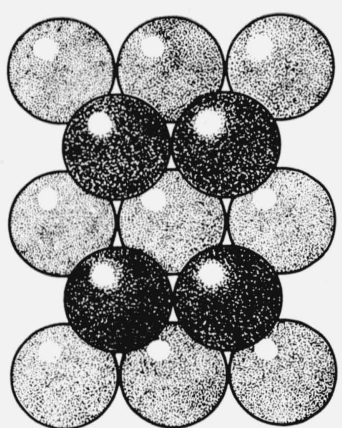

b
Figure 10. Sketches of packed spheres depicting the atomic arrangement in the dodecahedral plane of copper.

(a) The two top layers of atoms, and (b) the deposition of atoms of copper (dark spheres) in locations which perpetuate the orientation of the base metal, in kind.
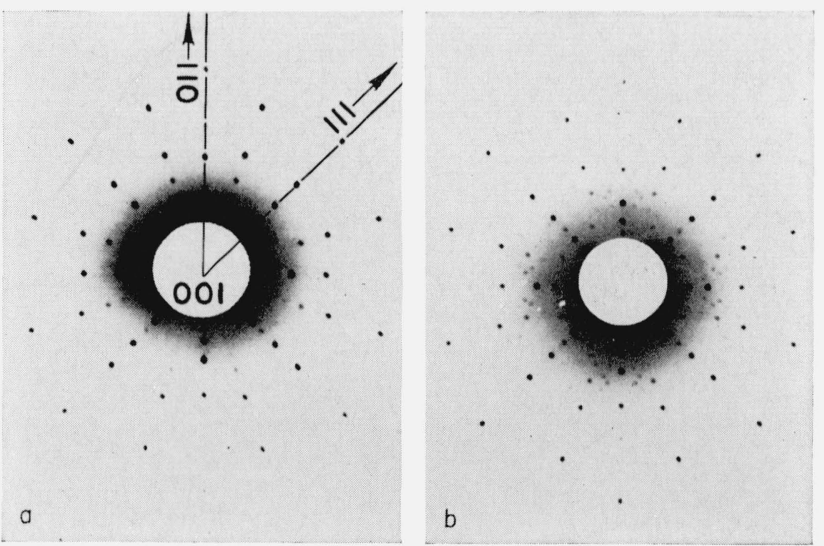

FIGURE 11. Laue back-reflection diffraction patterns.

(a) Before, and (b) after electrodeposition of copper on a copper monocrystal. The surface was parallel to a cubic plane and the X-ray beam normal to the surface.

spect to the uppermost two layers in order for the orientation of the base crystal to be continued in the electrodeposit. There is no position in the cubic surface, as such, in which an atom can locate that would not continue the orientation of the base crystal. The microstructure of the electrodeposit, figure 1 ,a, and the diffraction pattern, figure $11, b$, (with interpretation of extra spots as described herein), however, indicate that at some period during electrodeposition, situations are created whereby atoms are able to locate in a choice of positions, in one case where the atomic configuration of the base metal will be continued, in kind, and, in a second case, where the atomic arrangement is twinned with respect to the base metal.

It is postulated that the atoms at first all locate as shown in figure 14,b, building up as monolayers of atoms in accordance with the orientation of the base crystal as the atoms deposit laterally around the the atoms first depositing at random sites on the surface. In time, however, some of the layers of depositing atoms grow thicker than others as they grow laterally about nucleating atoms depositing at

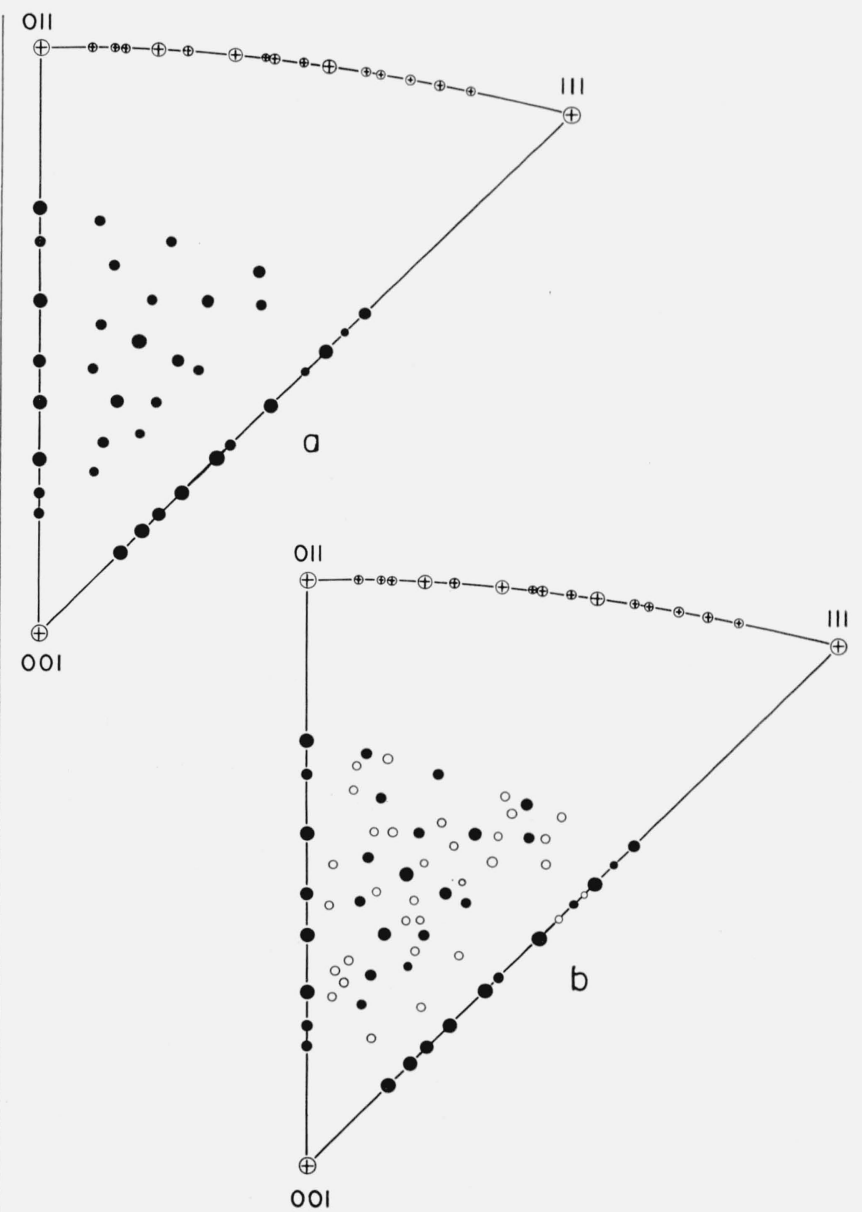

Figure 12. Unit stereographic triangles showing orientation of poles that correspond to the reflection spots in figure 11.

scattered points over the entire surface of the electrodeposit. This gives rise to the formation of octahedral planes as shown in figure $14, \mathrm{~b}$, on which planes subsequently depositing atoms can locate in a choice of position, as in the sketches shown in figure 5 . Atoms locating in twin positions on such surfaces, when viewed in a direction normal to the cubic plane, are depicted by the dark spheres in figure 14,c.

It is suggested that the atoms depositing from solution at low-current density have a great tendency to deposit such as to form monolayers whose orientation is the same as that of the base crystal. With increasing deposition time, twinning occurs on the octahedral planes, with a consequent formation of areas in which the atoms are in a state of misfit, these areas at first constituting twin boundaries. With additional deposition time the areas of misfit are aggravated and eventually become so large as to destroy the ability of depositing atoms to fit into the electrodeposit with any semblance of regularity, at which time a grain boundary is formed. Although there is considerable grain-boundary area in figure $1, \mathrm{a}$, the diffraction pattern, figure $11, \mathrm{~b}$, indicates that the microcrystals in the electrodeposit which have grown in accordance with the orientation of the base 


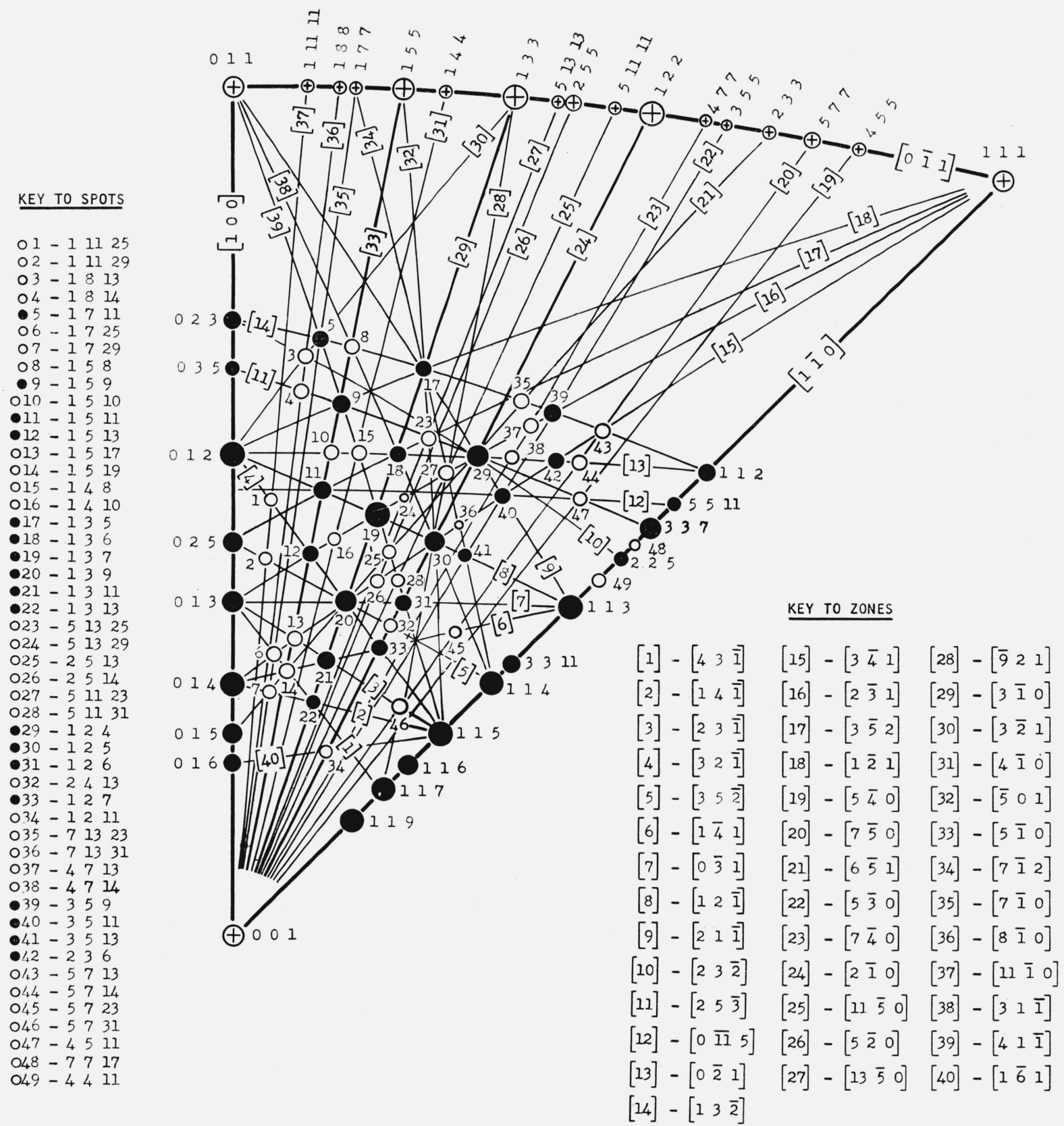

Figure 13. Stereographic triangle identifying spots and zones shown in figure 12.

crystal still predominate the structure. It is suggested that additional deposition would result in more and more areas becoming twinned and consequently the development of more areas of misfit as the preferentially oriented microcrystals grow into each other. Eventually, the areas of misfit evolve from twin boundaries to grain boundaries as the degree of misfit is further aggravated. The subsequent increase in the number of grain boundaries eventually diminishes the surfaces on which the atoms can preferentially locate and the entire surface then becomes a mass of microcrystals randomly oriented.

\section{Conclusions}

As a result of an analysis of Laue back-reflection diffraction patterns obtained from copper electrode deposits on monocrystalline copper base crystals of varying surface orientation at a current density of approximately $30 \mathrm{ma} / \mathrm{cm}^{2}$, using an acid-sulfate electrolyte, the following conclusions can be drawn:

1. A copper electrodeposit on a surface parallel to the dodecahedral plane is monocrystalline in character and duplicates the orientation of the base crystal.

2. Copper electrodeposited on a surface parallel to the octahedral plane has a very preferentially ori- 
TABLE 1. Apparent reflecting planes shown in figure 13 and calculated true reflecting planes present in the twinned portions of the electrodeposit.

\begin{tabular}{|c|c|c|c|c|c|}
\hline Spot & $\begin{array}{l}\text { Apparent } \\
\text { reflecting } \\
\text { plane }\end{array}$ & $\begin{array}{l}\text { True } \\
\text { reflecting } \\
\text { plane }\end{array}$ & Spot & $\begin{array}{l}\text { Apparent } \\
\text { reflecting } \\
\text { plane }\end{array}$ & $\begin{array}{l}\text { True } \\
\text { reflecting } \\
\text { plane }\end{array}$ \\
\hline $\begin{array}{l}1 \\
2 \\
3 \\
4 \\
6\end{array}$ & $\begin{array}{rrr}1 & 11 & 25 \\
1 & 11 & 29 \\
1 & 8 & 13 \\
1 & 8 & 14 \\
1 & 7 & 25\end{array}$ & $\begin{array}{lll}\overline{5} & \overline{3} & 7 \\
1 & 9 & 5 \\
\overline{3} & \overline{1} & 4 \\
0 & 5 & 2 \\
5 & \overline{1} & 7\end{array}$ & $\begin{array}{l}27 \\
28 \\
32 \\
34 \\
35\end{array}$ & $\begin{array}{rrrr}5 & 11 & 23 \\
5 & 11 & 31 \\
2 & 4 & 13 \\
1 & 2 & 11 \\
7 & 13 & 23\end{array}$ & $\begin{array}{ccc}\frac{5}{7} & 1 & 7 \\
7 & 7 & 5 \\
1 & 4 & 2 \\
1 & 3 & 2 \\
\overline{5} & 7 & 3\end{array}$ \\
\hline $\begin{array}{r}7 \\
8 \\
10 \\
13 \\
14\end{array}$ & $\begin{array}{rrr}1 & 7 & 29 \\
1 & 5 & 8 \\
1 & 5 & 10 \\
1 & 5 & 17 \\
1 & 5 & 19\end{array}$ & $\begin{array}{lll}\overline{7} & 5 & 5 \\
0 & 3 & 1 \\
\overline{2} & \overline{1} & 3 \\
1 & 5 & 3 \\
\overline{3} & \overline{3} & 5\end{array}$ & $\begin{array}{l}36 \\
37 \\
38 \\
43 \\
44\end{array}$ & $\begin{array}{rrr}7 & 13 & 31 \\
4 & 7 & 13 \\
4 & 7 & 14 \\
5 & 7 & 13 \\
5 & 7 & 14\end{array}$ & $\begin{array}{lll}7 & 1 & 9 \\
3 & 1 & 4 \\
3 & 4 & 2 \\
\frac{1}{2} & 5 & 1 \\
& 1 & 5\end{array}$ \\
\hline $\begin{array}{l}15 \\
16 \\
23 \\
24 \\
25\end{array}$ & $\begin{array}{rrr}1 & 4 & 8 \\
1 & 4 & 10 \\
5 & 13 & 25 \\
5 & 13 & 29 \\
2 & 5 & 13\end{array}$ & $\begin{array}{lll}\overline{2} & 2 & 1 \\
2 & 0 & 3 \\
1 & 9 & 3 \\
\overline{5} & 9 & 9 \\
\overline{3} & 3 & 2\end{array}$ & $\begin{array}{l}45 \\
46 \\
47 \\
48 \\
49\end{array}$ & $\begin{array}{lll}5 & 7 & 23 \\
5 & 7 & 31 \\
4 & 5 & 11 \\
7 & 7 & 17 \\
4 & 4 & 11\end{array}$ & $\begin{array}{lll}\overline{3} & \overline{3} & 7 \\
3 & 9 & 5 \\
1 & 4 & 1 \\
\overline{3} & 5 & 3 \\
\overline{2} & 3 & 2\end{array}$ \\
\hline 26 & $\begin{array}{llll}2 & 5 & 14\end{array}$ & 304 & & & \\
\hline
\end{tabular}

ented polycrystalline character, some of the individual crystals having the same orientation as the base crystal and some being twinned with respect to it.

3 . The copper electrodeposit on a surface parallel to a cubic plane is polycrystalline in character, the individual crystals being very preferentially oriented with respect to the base crystal, some continuing the base-crystal orientation and others being twinned with respect to it.
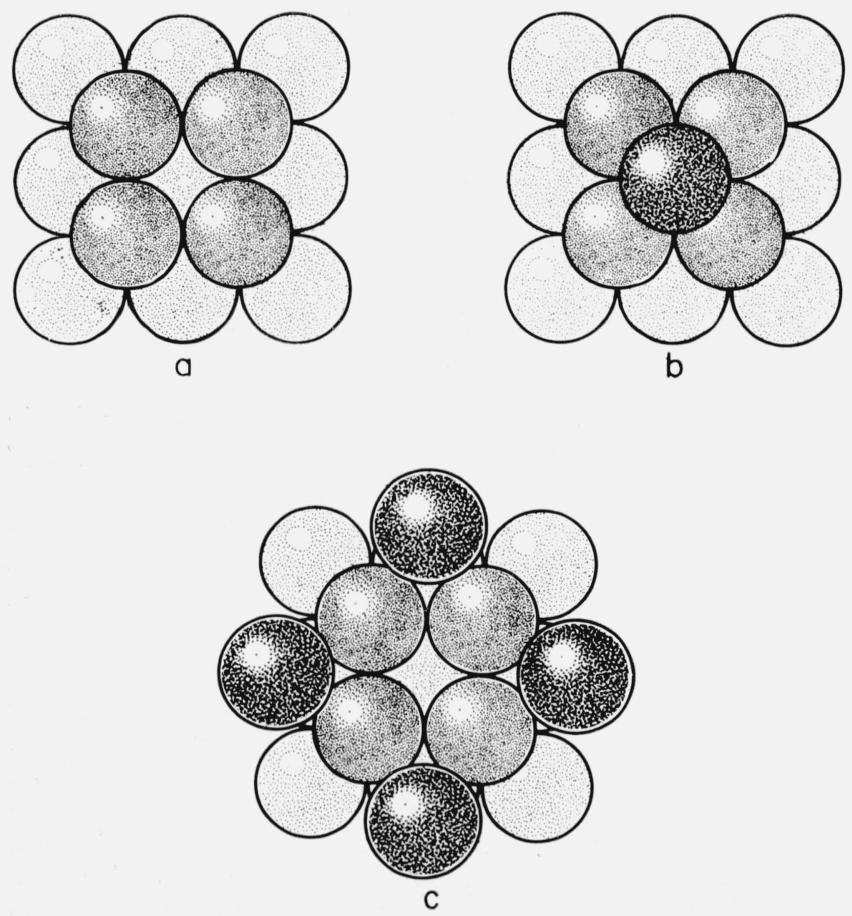

FIGURE 14. Sketches of packed spheres depicting the atomic arrangements in the cubic plane of copper.

(a) The two top layers of atoms, (b) the deposition of an atom of copper (dark sphere) in location which will perpetuate orientation of base metal in electrodeposit, in kind, and (c) the deposition of atoms of copper (dark spheres) in locations corresponding to the twin positions on the octahedral planes of the base metal.

\section{References}

[1] W. Blum and H. S. Rawdon, Trans. Am. Electrochem. Soc. 44, 305 (1923).

[2] H. Hirata and Y. Tanaka, Mem. Coll. Sci. Kyoto Imp. Univ. [A] 15, 9 (1932).

[3] W. A. Wood, Proc. Phys. Soc. (London) 43, 138 (1931).

[4] H. E. Farnsworth, Phys. Rev. 35, 1131 (1930).

[5] H. Leidheiser and A. T. Gwathmey, Trans. Electrochem. Soc. 91, 95 (1947).

[6] C. S. Barrett, Structure of metals, 2d ed., p. 513 (1952).

[7] L. E. Samuels, J. Inst. of Metals 85, 177 (1956-57).

[8] A. W. Hothersall, Trans. Faraday Soc. 31, 1242 (1935).

[9] W. Cochrane, Proc. Phys. Soc. (London) 48, 723 (1936).

[10] N. Takahasi, J. chim. phys. 50, 624 (1953).

\section{Appendix. Interpretation of Diffraction Patterns}

The procedures for indexing the open-circle spots shown in figures 3 , a and $12, \mathrm{~b}$ are outlined in the following sections.

\subsection{Electrodeposit on Surface Parallel to Octahedral Plane}

Figure 15 is a pole figure showing the relationship between principal poles in a stereographic projection when the octahedral pole is at the center of the pole figure. The darkly outlined triangle, 111 , 110 , 100 , is the stereographic triangle shown in figure 4 . It is one of six stereographic triangles bounded by the three cubic zones shown in the pole figure. The six stereographic triangles bounded by the short dashed lines are the above-mentioned six triangles rotated $60^{\circ}$ clockwise about the normal to $\left(\begin{array}{lll}1 & 1 & 1\end{array}\right)$. The poles shown as open circles and identified with italicized indices in the rotated triangles are thus in twin positions with respect to the basic orientation. Only that part of the basic projection bounded by the cubic zones is shown in the rotated position in order to minimize confusion. Also shown in the basic projection are several principal zones, $\left[\begin{array}{lll}1 & 0 & 1\end{array}\right]$,

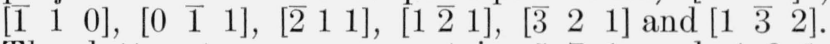
The latter two zones contain $577 \quad 1$ and 4221 , respectively, which are shown as solid circles as it is known that reflections from the planes normal to these two poles were present in the diffraction pattern from the electrodeposit on the surface parallel to the octahedral plane.

After rotating the basic projection $60^{\circ}$ clockwise about the normal to $\left(\begin{array}{lll}1 & 1 & 1\end{array}\right)$, zones $\left[\begin{array}{lll}1 & 0 & 1\end{array}\right]$ and $\left[\begin{array}{lll}\overline{1} & 1 & 0\end{array}\right]$ in the rotated projection will coincide with zones [ $\left[\begin{array}{lll}1 & 1 & 0\end{array}\right]$ and $\left[\begin{array}{lll}0 & 1 & 1\end{array}\right]$, respectively, in the basic projection. The indices of the rotated zones are shown in italics. By rotating one zone so that it coincides with another, all poles on the two zones will lie on one zone. Poles on different zones, which before rotation have equivalent distances from the axis of rotation, will, of course, coincide when the pertinent zones coincide after rotation. Thus, in figure 15 and in figure 4, all poles shown as solid circles in zone $\left[\begin{array}{lll}1 & 1 & 0\end{array}\right]$ will lie on zone $\left[\begin{array}{lll}0 & 1 & 1\end{array}\right]$, where they are shown as open circles, after $60^{\circ}$ clockwise rotation about the normal to $\left(\begin{array}{lll}1 & 1 & 1\end{array}\right)$. There is no open-circle spot corresponding to 771 


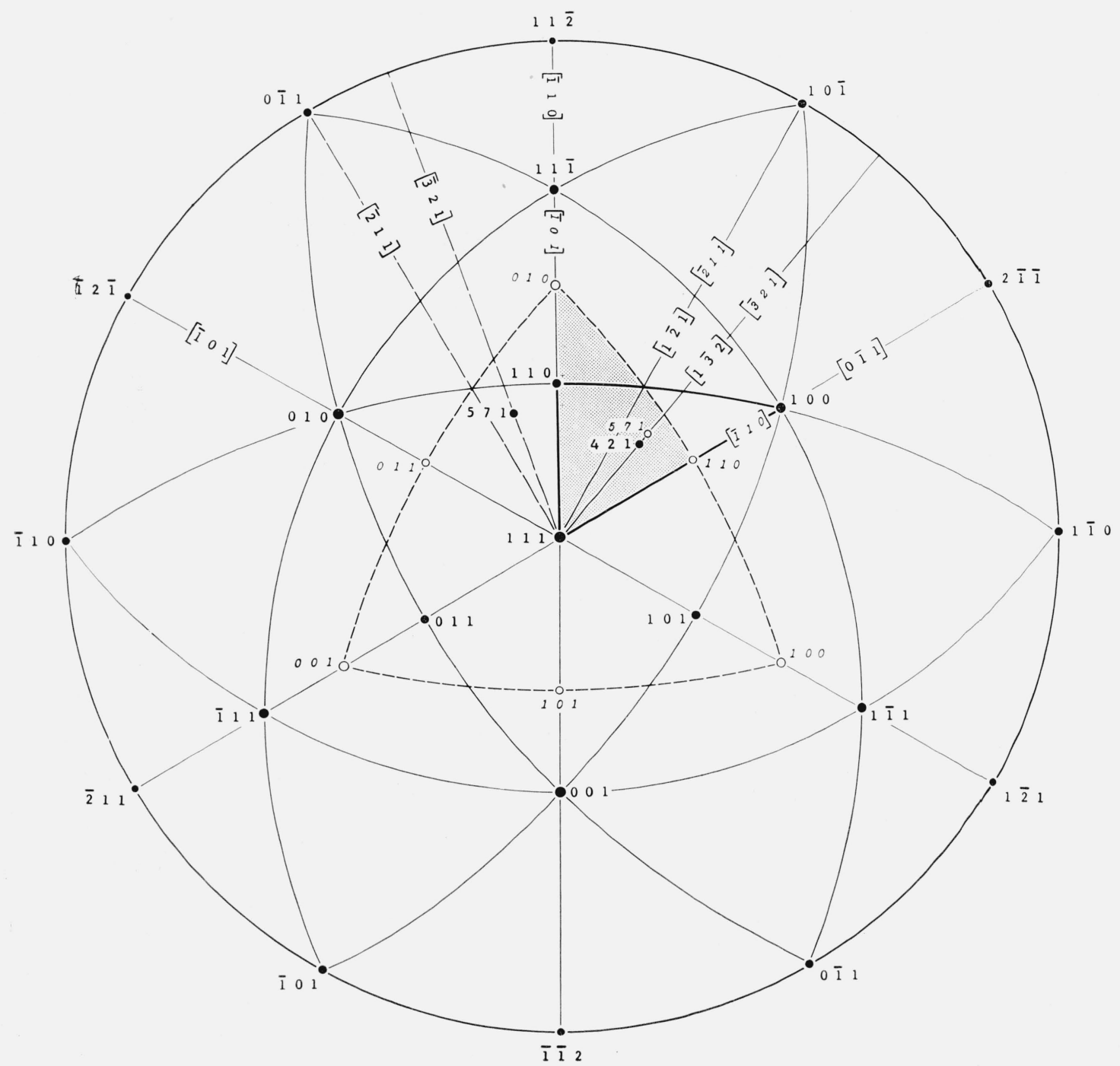

FiguRE 15. Stereographic projection showing orientation of base crystal and the 111 twin orientation.

Indices of planes and zones of the twin orientation are in italics and portions of the twin cubic zonal circles are dashed.

on zone $\left[\begin{array}{lll}0 & \overline{1} & 1\end{array}\right]$ in this case because $7 \quad 7 \quad 1$ and 311 both make equivalent angles, in their respective zones, with the axis of rotation, and, therefore, 771 is superimposed on 311 after rotation.

Similarly, in figure 15 , all poles on zone $\left[\begin{array}{lll}\overline{1} & 0 & 1\end{array}\right]$ will fall on zone [ $\left[\begin{array}{lll}1 & 1 & 0\end{array}\right]$ after rotation. This is shown in figure 4. Here again, we have the case of a pole on one zone superimposing on a pole in a second zone after rotation. 131 in zone [ $\left[\begin{array}{lll}1 & 0 & 1\end{array}\right]$ (not shown in figure 15) coincides, after rotation, with 771 in zone [ $\left[\begin{array}{lll}1 & 1 & 0\end{array}\right]$ (shown in figure 4) and is not, therefore, shown as an open-circle spot in figure 4.

In rotating the basic projection $60^{\circ}$ clockwise about the normal to $\left(\begin{array}{lll}1 & 1 & 1\end{array}\right)$, zone $\left[\begin{array}{lll}\overline{2} & 1 & 1\end{array}\right]$ will coincide with zone [ $\left[\begin{array}{lll}1 & \overline{2} & 1\end{array}\right]$ and, therefore, all poles in zone $\left[\begin{array}{lll}\overline{2} & 1 & 1\end{array}\right]$ will superimpose on the poles in zone $\left[\begin{array}{l}1 \\ 2\end{array}\right]$. It will be noted that all poles on zone $\left[\begin{array}{lll}1 & \overline{2} & 1\end{array}\right]$ in figure 4 are shown as closed circle spots. All zones of the type [ [ $\left.\begin{array}{lll}2 & 1 & 1\end{array}\right]$ and, consequently, all poles on those zones are common both to the basic projection and to the basic projection rotated $60^{\circ}$ clockwise about the normal to $\left(\begin{array}{lll}1 & 1 & 1\end{array}\right)$.

The indexing of all of the open-circle spots shown within the stereographic triangle, figure 4 , is accomplished in the same manner described above for indexing the open circle spots on the sides of the triangle, by the superimposition of zones of rotated triangles on zones of the basic projection. In nigure 15 the normal to $\left(\begin{array}{lll}5 & 7 & 1\end{array}\right)$ in the basic projection is shown within triangle $111,110,010$, in zone [ $\left.\begin{array}{lll}3 & 2 & 1\end{array}\right]$. When this particular triangle is rotated $60^{\circ}$ clockwise about the normal to $\left(\begin{array}{lll}1 & 1 & 1\end{array}\right)$, it is reoriented with respect to the basic projection as shown by the shaded triangle. In the rotated position, zone $\left[\begin{array}{lll}\overline{3} & 2 & 1\end{array}\right]$ now coincides with zone $\left[\begin{array}{lll}1 & \overline{3} & 2\end{array}\right]$ of the basic projection and the normal to $\left(\begin{array}{lll}5 & 7 & 1\end{array}\right)$, which lies on $\left[\begin{array}{lll}3 & 2 & 1\end{array}\right]$ of the basic projection, can be seen in relation to the normal to $\left(\begin{array}{lll}4 & 2 & 1\end{array}\right)$ of the basic projection. Compare with same spots in figure 4 . 


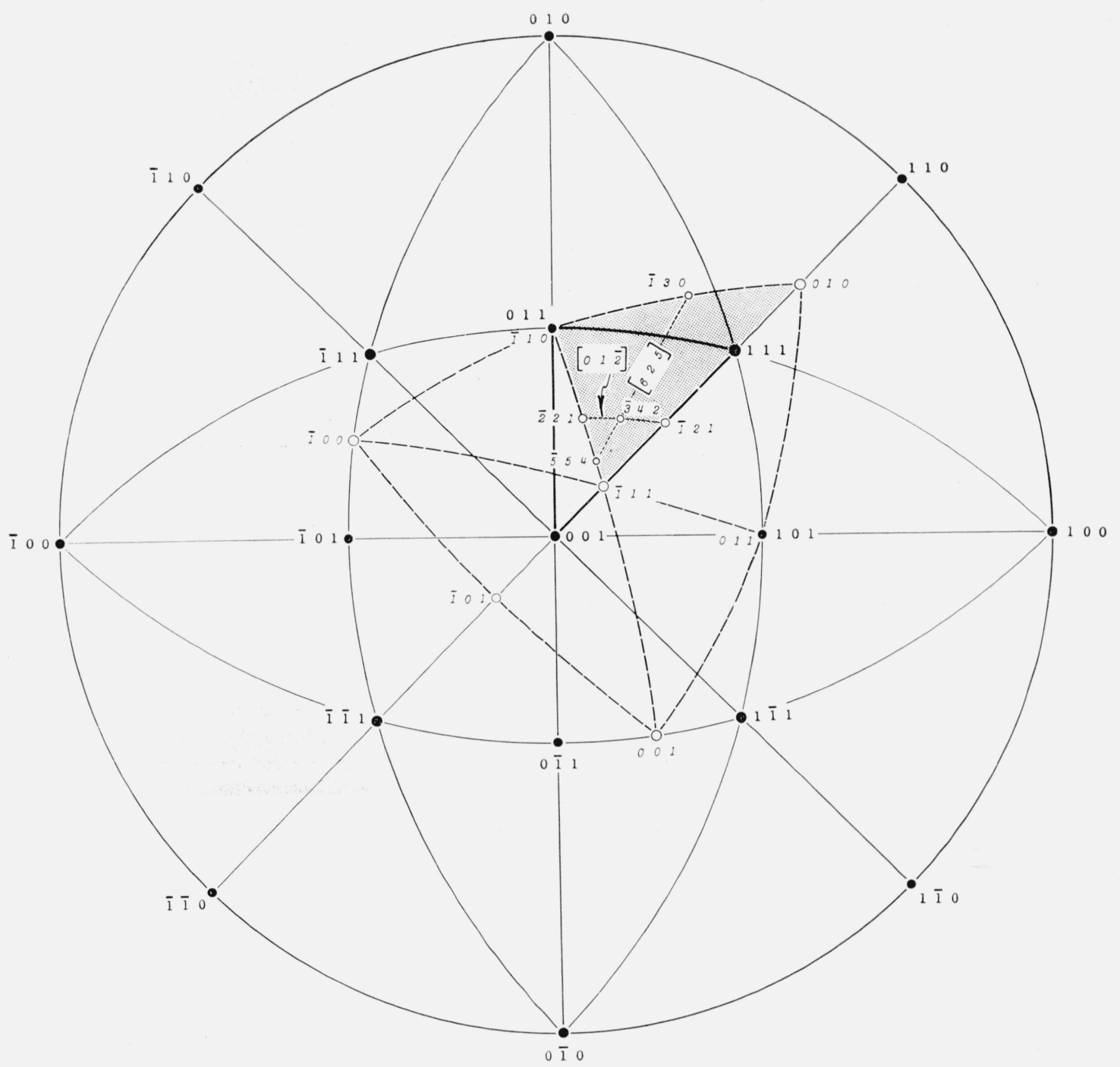

FiguRE 16. Stereographic projection showing method of reindexing reflections on basis of a twin orientation of the base crystal. Indices of the 111 twin orientation are shown in italies and parts of zonal circles are dashed.

All other open-circle spots within the stereographic triangle shown in figure 4 can be indexed in a similar manner.

The difference between the diffraction pattern in figure 2 , a and that in figure $2, b$ is the result of simple rotation about the surface normal, the rotational angle being $60^{\circ}$. This indicates that some of the microcrystals making up the electrodeposit on the surface parallel to the octahedral plane are twinned with respect to the base crystal.

\subsection{Electrodeposit on Surface Parallel to Cubic Plane}

The determination of the indices of the opencircle spots shown in figure $12, \mathrm{~b}$, which, when indexed with respect to the basic orientation, have the high indices shown in figure 13, can be accomplished by the following method:
Figure 16 is a pole figure showing the relationship between principal poles in a stereographic projection when the cubic pole is at the center of the pole figure. The darkly outlined triangle $111,0 \quad 11$, 001 , in the vicinity of the center of the pole figure is the stereographic triangle shown in figure 13.

An example of this method is illustrated by means of figure 16. In figure 13, spot number 38 is indexed 47 14. It is the spot (pole) common to zones $\left[\begin{array}{lll}0 & \overline{2} & 1\end{array}\right]$ and $\left[\begin{array}{lll}7 & \overline{4} & 0\end{array}\right]$ (see fig. 13) of the base crystal, the basic orientation of figure 16 . The dashed lines in figure 16 show the orientation of the octant 100 , $\begin{array}{lllllll}0 & 1 & 0,0 & 0 & 1 & \text { after a clockwise rotation of } 60^{\circ} \text { about }\end{array}$ the normal to the ( $\left.\begin{array}{lll}\overline{1} & \overline{1} & 1\end{array}\right)$. The rotated orientation of the $\overline{1} 11, \overline{1} 10,010$ unit triangle is shown by shading. All poles and zones in the rotated orientations are shown in italies to differentiate them from the corresponding poles and zones shown in the basic orientation. The two zones $\left[\begin{array}{lll}0 & \overline{2} & 1\end{array}\right]$ and $\left[\begin{array}{lll}7 & \overline{4} & 0\end{array}\right]$, re- 
indexed with respect to the system of indices of the rotated orientation should then define the indices of a spot that coincides with spot number 38, 4714 . The zone in the rotated orientation parallel to zone $\left[\begin{array}{lll}7 & \overline{4} & 0\end{array}\right]$ in the basic orientation, projected to intersect a zone in the former containing 010 and $\overline{1} 10$ intersects this zone at a point $26^{\circ} 34^{\prime}$ from $\overline{1} 10$. This is the angle between $\overline{1} 30$ and $\overline{1} 10$. The zone corresponding to $\left[\begin{array}{lll}7 & \overline{4} & 0\end{array}\right]$ also intersects a zone containing $\overline{1} 10$ and $\overline{1} 11$ in the rotated orientation at a distance $29^{\circ} 30^{\prime}$ from $\overline{1} 10$. This is the angle between $\overline{5} 54$ and $\overline{1} 10$. The normals to $\left(\begin{array}{lll}\overline{1} & 3 & 0\end{array}\right)$ and $(\overline{5} 54$ 4 $)$ then lie in a zone in the rotated orientation, which is parallel to zone $\left[\begin{array}{lll}7 & \overline{4} & 0\end{array}\right]$ in the basic orientation. This zone is thus indexed $\left[\begin{array}{lll}6 & 2 & 5\end{array}\right]$. The second zone in the rotated orientation, which is parallel to zone $\left[\begin{array}{lll}0 & \overline{2} & 1\end{array}\right]$ of the basic orientation, intersects a zone containing 010 and $\overline{1} 11$ in the rotated orientation at a point $19^{\circ} 28^{\prime}$ from $\overline{1} 11$. This is the angle between $\overline{1} 21$ and $\overline{1} 11$. The zone corresponding to $\left[\begin{array}{lll}0 & \overline{2} & 1\end{array}\right]$ also intersects a zone containing $\overline{1} 11$ and $\overline{1} 10$ in the rotated orientation at a point $15^{\circ} 48^{\prime}$ from $\overline{1} 11$. This is the angle between $\overline{2} 21$ and $\overline{1} 11$. The normals to $\left(\begin{array}{lll}\overline{1} & 2 & 1\end{array}\right)$ and $(\overline{2} \mathscr{2} 1)$ then lie in a zone of the rotated orientation, which is parallel to zone $\left[\begin{array}{lll}0 & \overline{2} & 1\end{array}\right]$ of the basic orientation. This zone is thus indexed $\left[\begin{array}{lll}0 & 1 & \overline{2}\end{array}\right]$. Zones $\left[\begin{array}{lll}6 & 2 & 5\end{array}\right]$ and $\left[\begin{array}{lll}0 & 1 & \overline{2}\end{array}\right]$ in the rotated orientation are then parallel to zones $\left[\begin{array}{lll}7 & \overline{4} & 0\end{array}\right]$ and $\left[\begin{array}{lll}0 & \overline{2} & 1\end{array}\right]$, re- spectively, of the basic orientation. The former two zones intersect to locate the normal to $(\overline{3} 42)$, which plane is thus parallel to high-index plane (4 7 14).

The above example was selected to illustrate the case where a clockwise rotation of $60^{\circ}$ about one of the four [ $\left.\begin{array}{lll}1 & 1 & 1\end{array}\right]$ axes resulted in low indices for the too high indices of the "apparent reflecting planes." A similar reindexing on the basis of rotations about the other three [ $\left[\begin{array}{lll}1 & 1 & 1\end{array}\right]$ axes resulted in high indices, too high to be accountable by the limitation imposed by the minimum wave length used in obtaining the diffraction patterns. By following the procedure used in the foregoing illustration, all of the apparent reflecting planes were reindexed and are listed in table 1 under the heading "True reflecting planes."

The author expresses his appreciation to G. A. Ellinger and H. C. Vacher for critical review of the manuscript and for suggestions regarding presentation of data; to Mr. Vacher for furnishing the copper monocrystal used in the investigation, and to R. J. Fallon, a summer staff member currently doing graduate work at Catholic University, for his industrious experimentation which, in addition to corroborating the results obtained by the author, furnished information that added materially to the author's concept of the growth of the electrodeposits, as described herein.

Washington, January 15, 1958. 